\title{
A Novel Predictive Model Associated with Osteosarcoma Metastasis
}

\author{
Han Zhang ${ }^{1,2}$ \\ Guanhong Chen ${ }^{2}$ \\ Xiajie Lyu ${ }^{3}$ \\ Chun Rong ${ }^{4}$ \\ Yingzhen Wang' \\ Ying $X u^{2}$ \\ Chengyu Lyu'
}

'Department of Orthopaedics, The Affiliated Hospital of Qingdao University, Qingdao City, Shandong Province, People's Republic of China; ${ }^{2}$ Department of Orthopaedics, Shanxian Central Hospital, Heze City, Shandong Province, People's Republic of China; ${ }^{3}$ Weifang Medical University, Weifang, Shandong, People's Republic of China; ${ }^{4}$ Department of Operation Room, The Affiliated Hospital of Qingdao University, Qingdao City, Shandong Province, People's Republic of China
Correspondence: Chengyu Lyu Email18266390I38@I63.com
Purpose: Long non-coding RNAs (lncRNAs) have diverse roles in modulating gene expression on both transcriptional and translational levels, but their involvement in osteosarcoma (OS) metastasis remains unknown.

Patients and Methods: Transcriptional and clinical data were downloaded from TARGET datasets. A total of seven lncRNAs screened by univariate cox regression, lasso regression, and multivariate cox regression analysis were used to establish the OS metastasis model. The area under the receiver operating characteristic curve (AUC) was used to evaluate the model. Results: The established model showed exceptional predictive performance ( 1 year: AUC = $0.92,95 \% \mathrm{Cl}=0.83-0.99 ; 3$ years: $\mathrm{AUC}=0.87,95 \% \mathrm{Cl}=0.79-0.96 ; 5$ years: $\mathrm{AUC}=0.86$, $95 \% \mathrm{Cl}=0.76-0.96)$. Patients in the high group had a poor survival outcome than those in the low group $(p<0.0001)$. GSEA analysis revealed that "NOTCH_SIGNALING" and "WNT_BETA_CATENIN_SIGNALING" were significantly enriched and that resting dendritic cells were associated with AL512422.1, AL357507.1, and AC006033.2 ( $<<0.05$ ).

Conclusion: Based on seven prognosis-related lncRNAs, we constructed a novel model with high reliability and accuracy for predicting metastasis in OS patients.

Keywords: lncRNAs, osteosarcoma, tumor metastasis, prognosis

\section{Introduction}

OS is the most common primary malignant bone tumor ${ }^{1}$ with a bimodal age distribution. The first peak occurs during adolescence, while the second peak occurs later in life. ${ }^{2-6}$ With the advancements of treatments, surgery and intensive multiagent chemotherapy became the mainstream strategies. ${ }^{7-9}$ The high incidence of metastasis in OS, particularly lung metastasis, results in a poor prognosis. ${ }^{10-12}$ The 5 -year overall survival rate for non-metastatic OS is about $75 \%$; while it is only $20 \%$ in metastatic patients. ${ }^{13,14}$ Thus, developing biomarkers for early detection and treatment is essential.

LncRNA is a non-coding RNA ranging length from 200 nucleotides (nt) to more than 100 kilobases $(\mathrm{kb}) .^{15}$ LncRNA modulates gene expression on both transcriptional and translational levels, including chromosomal rearrangement, histone modification, alternative splicing formation, and RNA stabilization. ${ }^{16}$ LncRNA has been found to play a role in tumor differentiation, invasion, and metastasis. ${ }^{17-21}$ LncRNA is implicated in the onset and progression of cancer metastasis. MALAT1, for example, primarily regulates lung cancer metastasis through epithelialmesenchymal transition (EMT). ${ }^{22,23}$ It has also been shown to promote metastasis in bladder cancer by activating Wnt signaling. ${ }^{24,25}$ HOTAIR promotes pancreatic cancer metastasis by suppressing interferon-related genes. ${ }^{26}$ MEG3 plays a decisive 
role in meningioma and gastric cancer by regulating autophagy and DNA repair. ${ }^{27,28}$ However, the role of lncRNA in OS remains unclear, and the IncRNA-based prediction model is currently lacking.

Advances in high-throughput technologies allow researchers to identify biomarkers that can be used to construct prediction models for personalized cancer treatment. In this study, we presented a new risk-score model for predicting the metastasis of OS patients based on lncRNAs. In addition, we would detect the critical biological process, immune-related cells, and immune checkpoint between high and low risk group.

\section{Materials and Methods}

\section{Target Data and Processing}

The clinical information and expression profiles, including mRNA and lncRNA expression profiles, were downloaded from the TARGET datasets (https://ocg.cancer.gov/programs/

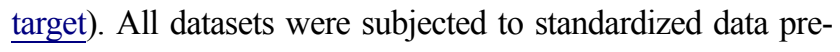
processing. In this study, a total of 22 metastasis and 66 primary OS tissue-related data were analyzed. Data preprocessing was performed using RStudio and included background correction, normalization, and batch effect adjustment.

\section{Feature Selection and Construction of Prognostic Model}

The Limma R package was used to compare differential gene expression of lncRNA and mRNA in 22 metastasis and 66 primary OS patients. $\mathrm{P}$-value $<0.05$ and $\mid \log 2$ (fold change [FC]) $\mid>1$ were set as the threshold criteria. Differentially expressed lncRNA in this study, were screened by univariate cox regression, least absolute shrinkage, and selection operator (LASSO) regression, and multivariate cox regression analysis in survival and glmnet $\mathrm{R}$ packages. The lncRNAbased prognosis risk score was established using a linear formula with the expression level multiplied regression model $(\beta)$. The median risk score was used to categorize patients into the high-risk group and low-risk group. The survival and survival ROC R packages were used to plot Kaplan-Meier (KM) survival and receiver operating characteristic (ROC) curves.

\section{Scale-Free Co-Expression Network and Enrichment Analysis}

Prognosis-related lncRNAs and metastasis-related mRNA were used to reconstruct the scale-free co-expression network. A total of 24 metastasis-related mRNAs associated with 7 metastasis-related lncRNAs (correlation coefficient $\mathrm{R}>0.3, \mathrm{p}<0.001$ ) were selected. Cytoscape software was used for network visualization. Using DAVID, we investigated the interactions between 24 metastasis-related mRNA and transcription factors (TF). GO pathway enrichment analyses were performed using the $\mathrm{R}$ package clusterProfiler. $\mathrm{P}<0.05$ was considered as used as the cutoff for statistical significance.

\section{Gene Set Enrichment Analysis and Gene Set Variation Analysis}

GSEA2.2.4 software was used to perform gene set enrichment analysis (GSEA) and generate an enrichment score. The gene sets used for the enrichment analysis were downloaded from the Molecular Signatures Database (MsigDB). After 1000 permutations, gene sets with a false discovery rate (FDR) less than 0.05 were reported to be significantly enriched. Gene Set Variation Analysis (GSVA) is a method for estimating the variation of gene set enrichment using samples from an expression data set. GSVA was performed using the Hallmark gene set in the GSVA package (from R Project 3.5.1) of R software with default parameters.

\section{Infiltrating Immune Cells}

The CIBERSORT algorithm (http://cibersort.stanford.edu), a deconvolution algorithm based on gene expression profiles, was used to evaluate the 22 different types of tumorinfiltrating immune cells in the metastatic and primary OS group. CIBERSORT can assess the composition of tumorinfiltrating immune cells, by using P-value as a criterion for measuring confidence in findings. The relationship between 7 prognosis-related lncRNAs and tumorinfiltrating immune cells was also investigated.

\section{Cell Lines and qPCR}

Highly metastasis human OS cell lines (MNNG) and weakly metastasis human OS cell lines (U2S2, SAOS-2, SJSA-1, and HOS) were purchased from iCell (Shanghai, China). Trizol reagent (Invitrogen Life Technologies) was used to isolate total RNA. Approximately $2 \mu \mathrm{g}$ RNA was reverse-transcribed into complementary DNA following the manufacturer's instructions and normalized using glyceraldehyde 3-phosphate dehydrogenase mRNA levels. Reverse-transcription quantitative PCR assay with PrimeScript RT Master Mix (Takara) was performed on a QuantStudio6 Flex real-time PCR system (Applied 
Biosystems). The following were the PCR amplification conditions: $2 \mathrm{~min}$ at $95^{\circ} \mathrm{C}$ for one cycle, followed by denaturation for $15 \mathrm{sec}$ at $95^{\circ} \mathrm{C}$, and extension for $60 \mathrm{sec}$ at $60^{\circ} \mathrm{C}$ for 38 cycles. Table $\mathrm{S} 1$ presents the primers used.

\section{Result}

\section{Differential Gene and IncRNA Screening}

This study included 22 metastatic and 66 primary OS samples. The Limma R package was used to select differentially expressed genes and lncRNA. As a result, 135 differentially expressed lncRNAs were identified, including 79 upregulated and 56 downregulated lncRNAs (Figure 1A). In addition, there were 171 differentially expressed genes (Figure 1B).

\section{Construction of Prognosis Assessment Model}

The findings from the univariate Cox regression analysis of 135 lncRNAs were used in LASSO regression to identify potential markers. Multivariate cox regression analysis identified seven prognosis-related lncRNAs (Figure 2A-C), including AL512422.1, AL008718.3, C5orf66-AS1, AL360182.2, CEBPA-DT, AC006033.2, and AL357507.1. All of these lncRNAs were used to establish the prognosis model, based on the following formula, $y=2.603^{*}$ AL512422.1+3.735*AL008718.3+18.047*C5orf66-AS1 $+1.292 *$ AL360182.2+0.475*CEBPA-DT+0.038*AC0060

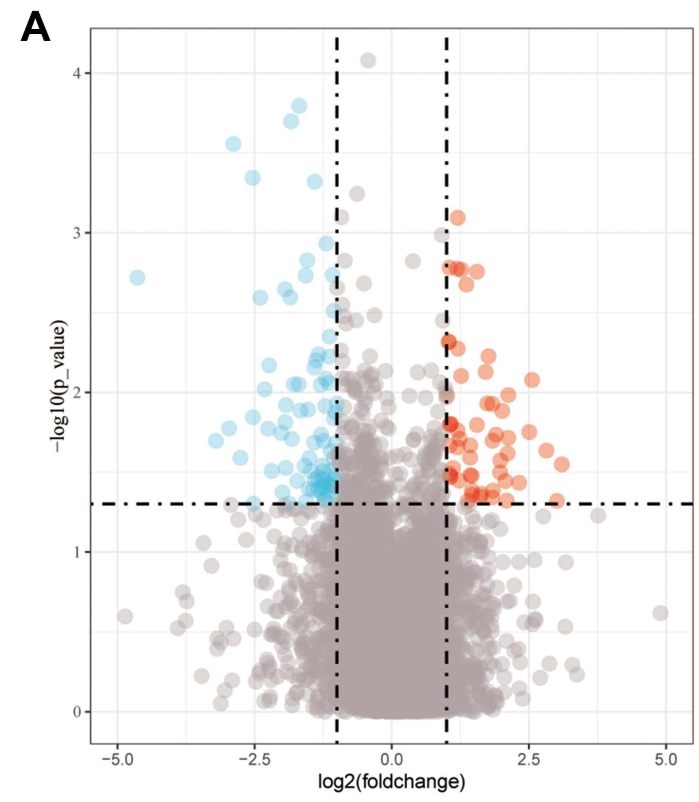

33.2+1.812*AL357507.1. The patients were divided into high-risk and low-risk groups based on the median risk score calculated using the above formula. Figure 2D depicts the expression of seven prognosis-related lncRNAs in high and low-risk groups. Moreover, the model had higher prediction ability, with all area under the curve (AUC) values $>0.8$ ( 1 year: $\mathrm{AUC}=0.92,95 \% \mathrm{Cl}=0.83-0.99 ; 3$ years: $\mathrm{AUC}=0.87,95 \% \mathrm{Cl}=0.79-0.96$; and 5 years: $\mathrm{AUC}=0.86$, 95\% $\mathrm{Cl}=0.76-0.96$ ) (Figure 2D). According to the $\mathrm{KM}$ survival curves (Figure 2D), the high-risk group had significantly poorer overall survival time than the low-risk group $(\mathrm{p}<0.0001, \mathrm{HR}=1.09,95 \% \mathrm{CI}=1.06-1.13)$. In addition, the KM survival curves indicated that five lncRNAs, including AL512422.1, AL008718.3, C5orf66-AS1, AL360182.2, and AL357507.1, were associated with poor overall survival. CEBPA-DT and AC006033.2, on the other hand, were associated with improved survival (Figure $3 \mathrm{~A}-\mathrm{G}$ ).

\section{Co-Expression Network and Enrichment Analysis}

To explore the mRNAs associated with the modelrelated lncRNAs and their potential TF, we integrated the lncRNA and gene data to construct a co-expression network. The metastasis-related genes that were closely associated with the seven prognosis-related lncRNAs were selected $(\mathrm{R}>0.3, \mathrm{p}<0.001)$. A total of 6 prognosisrelated lncRNAs and 24 metastasis-related genes were

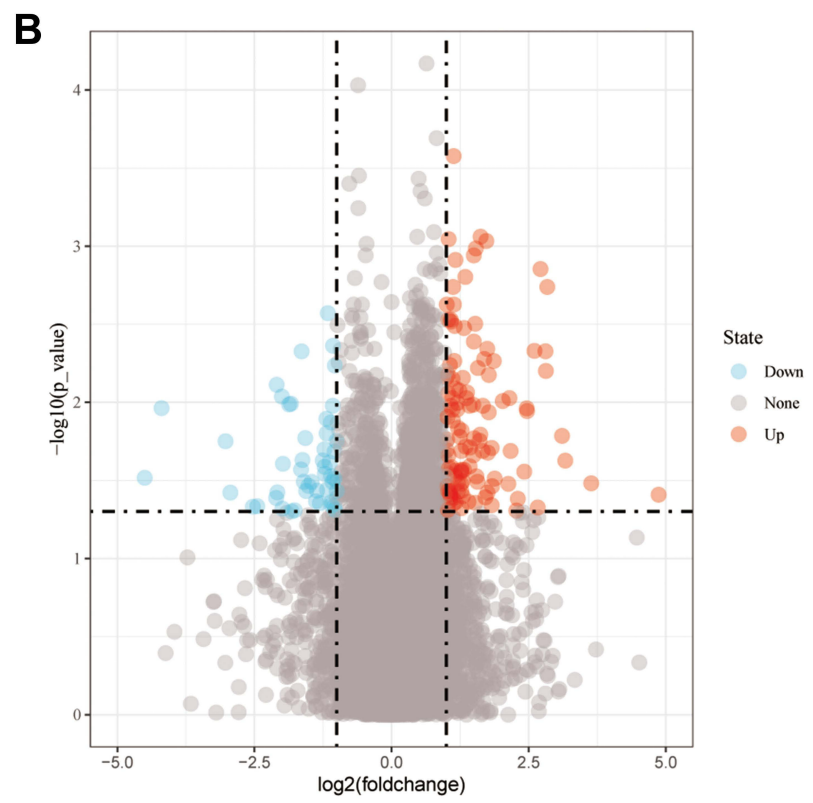

Figure I Differential expression profiles. (A) Differential IncRNA between metastatic and non-metastatic OS; (B) Differential mRNA between metastatic and nonmetastatic OS. 


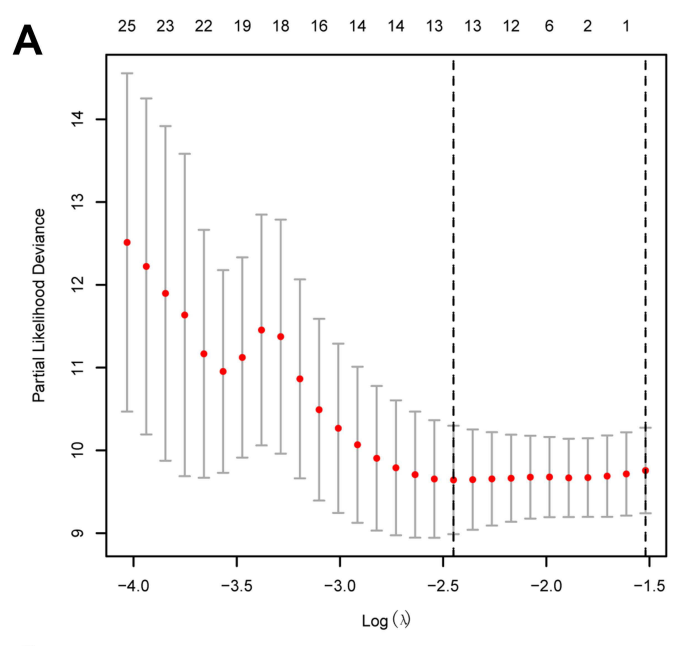

C

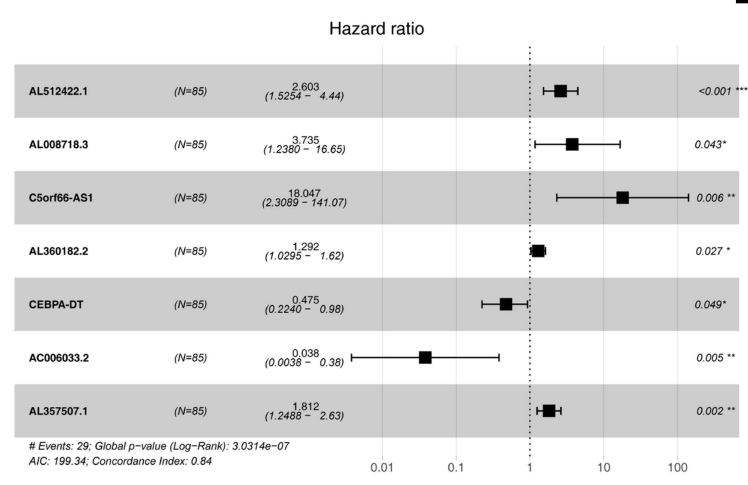

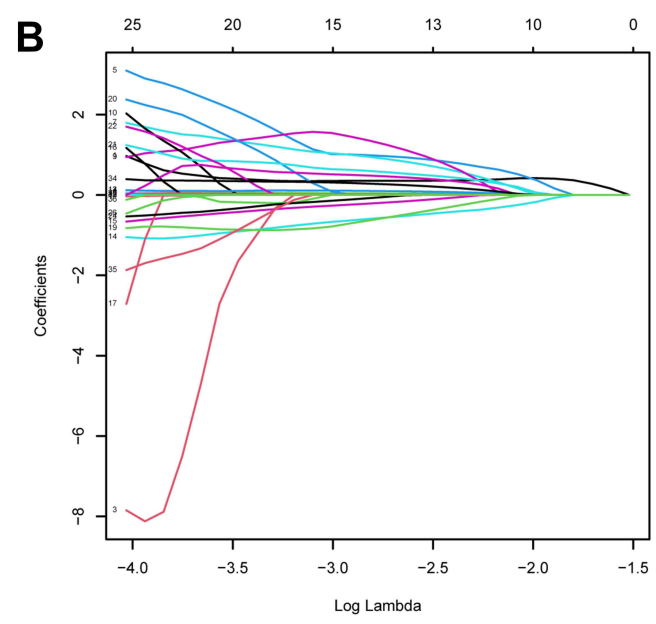

D

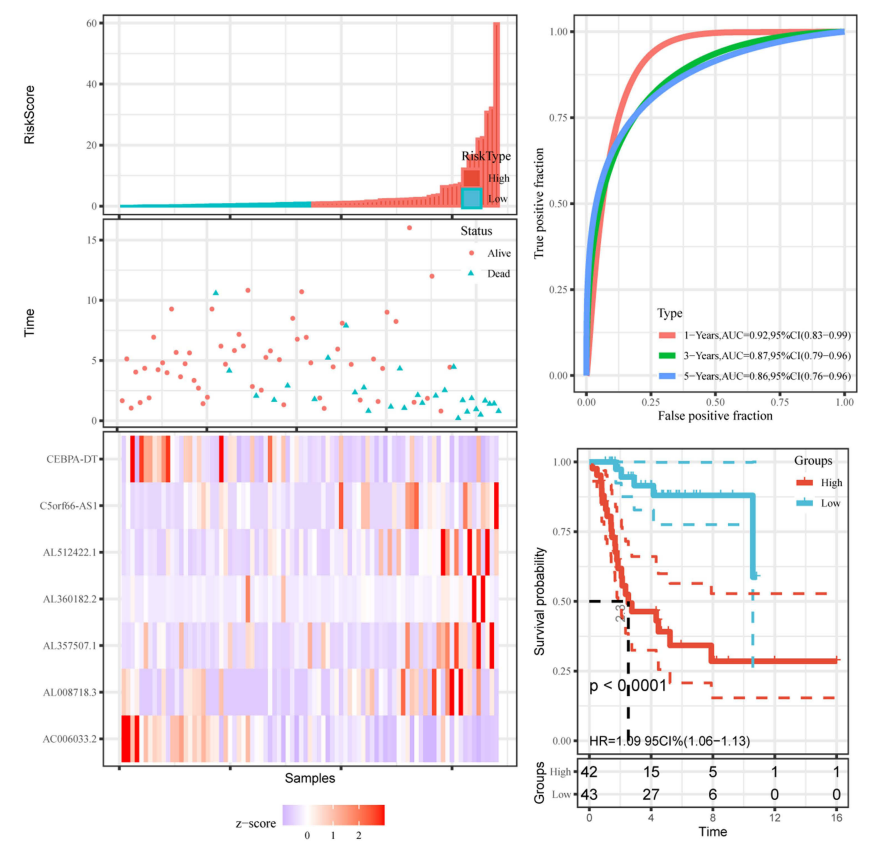

Figure 2 Identification of the prognosis-related IncRNA. (A and B) LASSO coefficient profiles; (C) multivariate cox analysis of seven model IncRNAs; (D) the risk scores, ROC curve, and Kaplan-Meier curve of patients.

used to construct the co-expression network (Figure 4A). AC006033.2 and AL512422.1 were the most important nodes. To further understand the functions of these genes, we assessed the interactions between 24 metastasis-related genes and 13 TFs (Figure 4B, Table 1). GO analysis was then carried out to determine the functional enrichment of the nodes in this co-expression network (Figure 4C). Several biological processes (BP) and cellular component (CC) pathways were found to be significantly enriched. According to BP, the nodes were mainly enriched in "catecholamine metabolic process", "lymphocyte proliferation", "mononuclear cell proliferation", "lymphocyte differentiation" and "leukocyte proliferation", while for CC they were enriched in, "integral component of synaptic membrane", "intrinsic component of synaptic membrane", and "glutamatergic synapse".

\section{GSVA Analysis of Seven Prognosis-Related IncRNAs}

We performed GSVA to figure out the dynamics of biological processes and pathways for Hallmark gene sets based on seven prognosis-related lncRNAs. As Figure 5 showed, "G2M_CHECKPOINT", "DNA_REPAIR" and "PI3K_AKT_MTOR_SIGNALING" were remarkably activated in the high AL512422.1 group, while the low AL512422.1 group was enriched in "WNT_BETA_ 
A $\quad$ AL512422.1
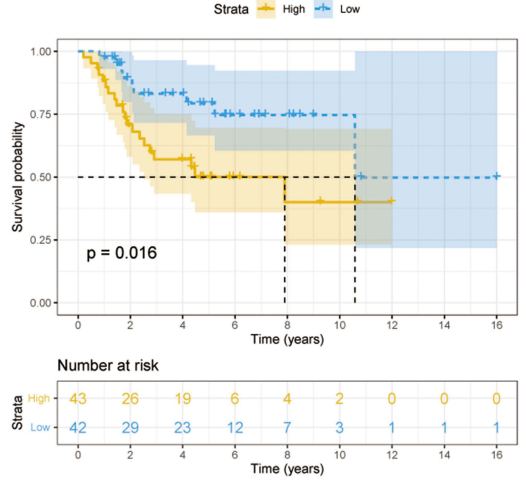

D
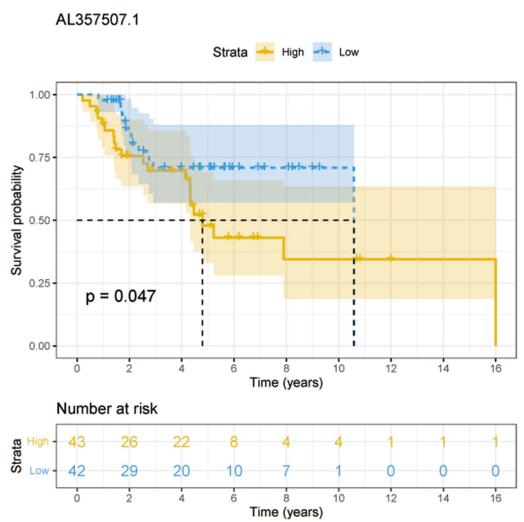

G

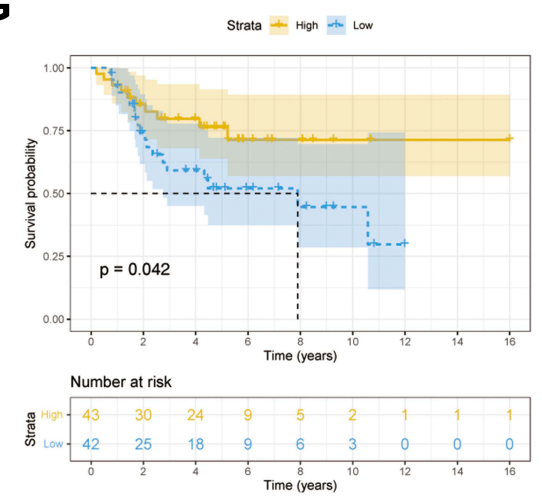

B AL008718.3
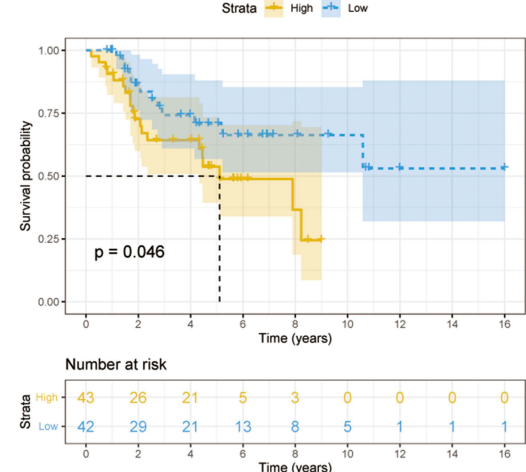

E $\quad$ AL360182.2
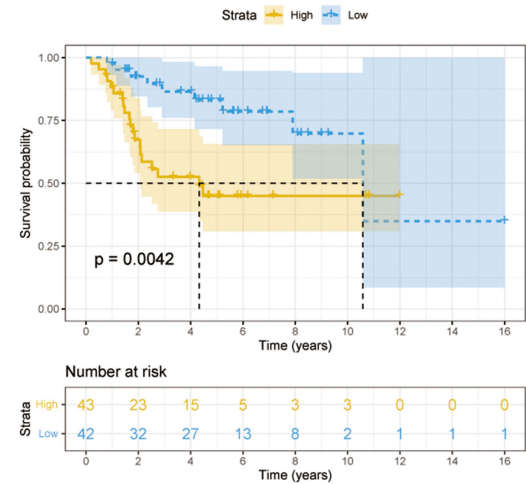

C ${ }^{A C 000633.2}$
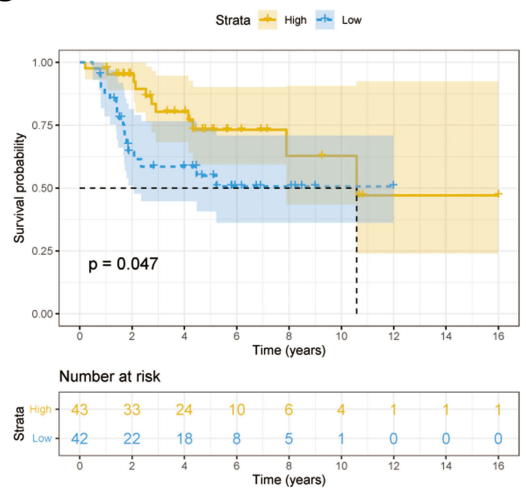

F ${ }^{C 50 \mathrm{r} 66-\mathrm{AS} 1}$
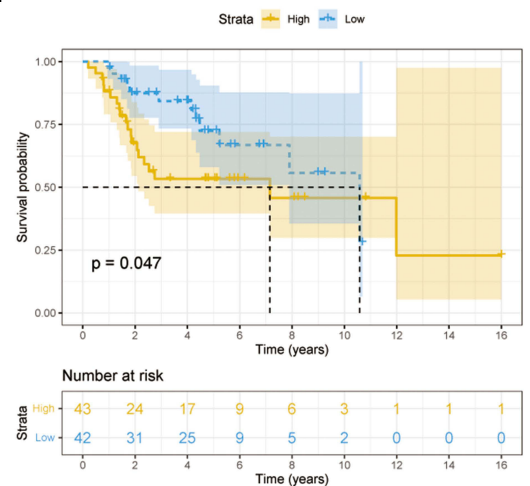

Figure 3 Kaplan-Meier curve of seven prognosis-related IncRNAs. (A) AL5I2422.I. (B) AL0087I8.3. (C) AC006033.2. (D) AL357507.I. (E) AL360I82.2. (F) C5orf66-ASI. (G) CEBPA-DT.

CATENIN_SIGNALING" and "KRAS_SIGNALING_ DN". Upregulation of AL008718.3 activated "WNT BETA_CATENIN_SIGNALING" and "TGF_BETA_ SIGNALING", but downregulation of AL008718.3 led to "HEDGEHOG_SIGNALING" and "PI3K_AKT MTOR_SIGNALING". In addition, AC006033.2 high level was significantly enriched for "HYPOXIA", "APOPTOSIS" and "NOTCH_SIGNALING", whereas low level triggered "DNA_REPAIR" and "G2M CHECKPOINT" process. Notably, "GLYCOLYSIS" and "NOTCH_SIGNALING" were all enriched in
AL357507.1 and AL360182.2 low groups. For the C5orf66-AS1 low group, "TGF_BETA_SIGNALING", "HYPOXIA" and "NOTCH_SIGNALING" were enriched. The C5orf66-AS1 high group is related to "WNT_BETA_CATENIN_SIGNALING". At last, we observed enrichment of "G2M_CHECKPOINT", "DNA_ REPAIR" and "PI3K_AKT_MTOR_SIGNALING" in the CEBPA-DT high group and "WNT_BETA CATENIN_SIGNALING" in the low group. The top high-frequency enriched pathways were "NOTCH_SIGNALING", "HYPOXIA", "WNT_BETA_ 

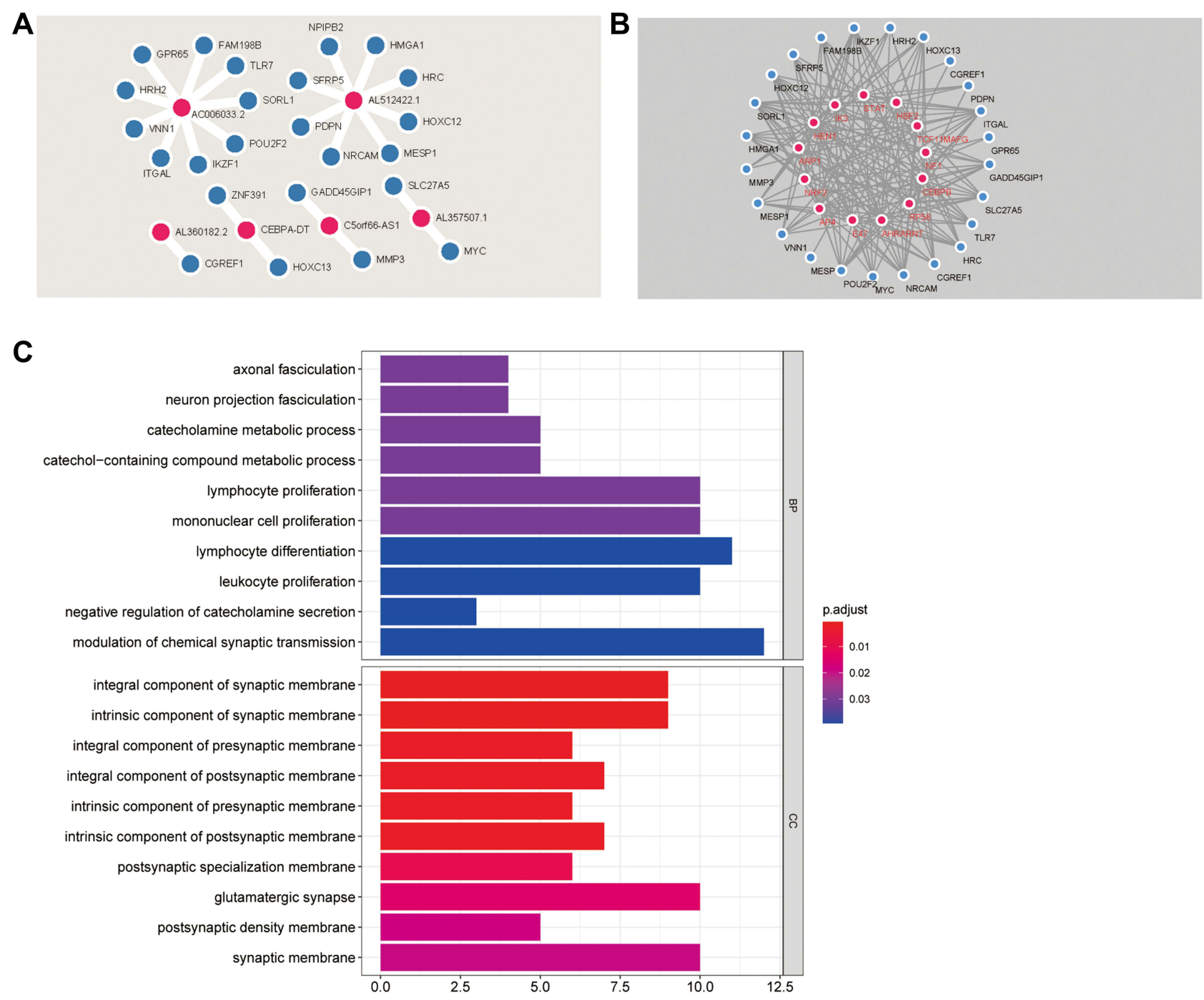

Figure 4 Co-expression network and enrichment analysis. (A) Co-expression network of IncRNA and mRNA; (B) Co-expression network of mRNA and TF; (C) GO analysis of the genes in co-expression network.

CATENIN_SIGNALING" and "HEDGEHOG_SIGNA LING".

\section{Correlation Between Prognosis-Related IncRNAs and Infiltrating Immunocyte Fractions}

To investigate the differences between the primary and metastatic OS, 22 infiltrating immunocyte fractions were compared. As shown in Figure 6A, the difference between the two groups was insignificant. Then, the relationship between seven prognosis-related lncRNAs and 22 infiltrating immune cells was analyzed (Figure 6B). AC006033.2 was found to have the strongest relationship with resting dendritic cells, macrophages M0, macrophages M2, activated mast cells, activated neutrophils, and memory $\mathrm{T}$ cells
CD4. AL357507.1 had a close relationship with dendritic cells resting, macrophages M1, neutrophils and plasma cells. CEBPA-DT was associated with monocyte, T cells CD4 naïve, $\mathrm{T}$ cells CD8 and T cells regulatory (Tregs). C5orf66-AS1 and AL512422.1 were only related to dendritic cells activated and dendritic cells resting, respectively. On the contrary, for AL360182.2 and AL008718.3, there was no association with immune cells.

\section{mRNA Expression of Seven IncRNAs in OS Cell Lines}

The mRNA level of seven lncRNAs (AL512422.1, AL008718.3, C5orf66-AS1, AL360182.2, CEBPA-DT, AC006033.2, and AL357507.1) in five OS cell lines were examined. MNNG cell line was found to have 


\begin{tabular}{|c|c|c|c|c|c|c|c|c|}
\hline 愛 & $\begin{array}{l}\hat{n} \\
\text { مी } \\
\text { o. } \\
\text { o. }\end{array}$ & $\begin{array}{l}\hat{\hat{n}} \\
\text { On } \\
\text { o. }\end{array}$ & 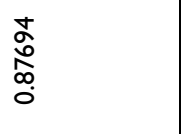 & $\begin{array}{l}\text { पे } \\
\stackrel{0}{0} \\
\text { o. } \\
0\end{array}$ & 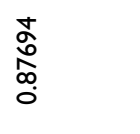 & $\begin{array}{l}\bar{ָ} \\
\text { مू } \\
\text { o. }\end{array}$ & 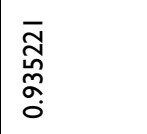 & 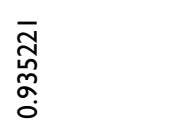 \\
\hline 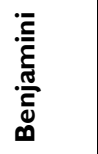 & $\begin{array}{l}\hat{n} \\
\text { مी } \\
\text { o. } \\
0\end{array}$ & $\begin{array}{l}\hat{n} \\
\frac{\hat{n}}{0} \\
0 \\
0\end{array}$ & $\begin{array}{l}\text { t. } \\
\stackrel{0}{0} \\
\text { कo } \\
0\end{array}$ & $\begin{array}{l}\text { ¿े } \\
\stackrel{0}{0} \\
\text { o. } \\
0\end{array}$ & $\begin{array}{l}\text { ¿े } \\
\text { ô } \\
\text { कo } \\
\text { o. }\end{array}$ & 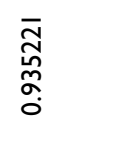 & $\begin{array}{l}\bar{ָ} \\
\text { مू } \\
\text { o. }\end{array}$ & $\begin{array}{l}\overline{\mathcal{N}} \\
\text { مू } \\
\text { o. }\end{array}$ \\
\hline 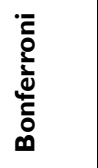 & $\begin{array}{l}\bar{\varpi} \\
\text { స్ర్ర } \\
\text { ○े }\end{array}$ & $\begin{array}{l}\hat{\infty} \\
o \\
o \\
o \\
0\end{array}$ & 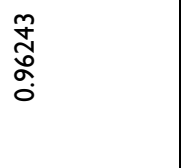 & 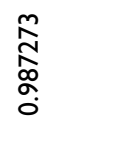 & $\begin{array}{l}\overline{\bar{\sim}} \\
\stackrel{\infty}{\infty} \\
\stackrel{0}{0}\end{array}$ & $\begin{array}{l}\frac{1}{\circ} \\
\text { o } \\
0 \\
0\end{array}$ & $\begin{array}{l}\alpha \\
\alpha \\
\alpha \\
\alpha \\
\sigma \\
\alpha\end{array}$ & $\begin{array}{l}\text { ڤ̊ } \\
\text { مू } \\
\text { o. }\end{array}$ \\
\hline 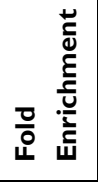 & 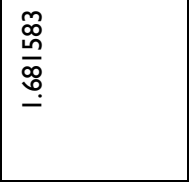 & 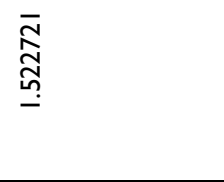 & 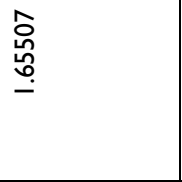 & 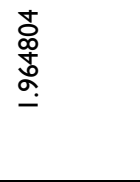 & 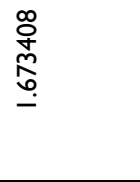 & 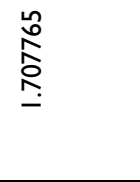 & 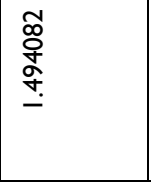 & $\frac{\frac{\tilde{\sigma}}{\sigma}}{\frac{\sigma}{\underline{t}}}$ \\
\hline ஃ ஃ & 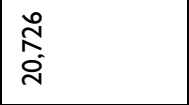 & 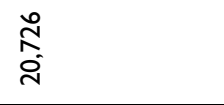 & 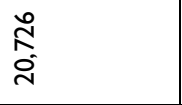 & $\begin{array}{l}\text { స̃ } \\
\text { ô. }\end{array}$ & $\begin{array}{l}\text { N̦ } \\
\text { ồ }\end{array}$ & $\begin{array}{l}\stackrel{2}{N} \\
\text { î }\end{array}$ & 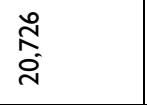 & $\begin{array}{l}\stackrel{2}{N} \\
\text { î. }\end{array}$ \\
\hline$\stackrel{0}{\circ}$ & $\frac{0}{a}$ & 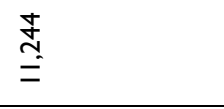 & $\frac{\hat{\sigma}}{\infty}$ & 卓 & ⿳八人 & ల్ల్ & ईे & $\frac{8}{\underline{0}}$ \\
\hline 苞 & $\ddot{\sim}$ & $\ddot{\sim}$ & $\ddot{\sim}$ & $\ddot{\sim}$ & $\ddot{\sim}$ & $\ddot{\sim}$ & $\ddot{\sim}$ & $\ddot{\sim}$ \\
\hline $\begin{array}{l}\stackrel{y}{ \pm} \\
\stackrel{\Xi}{ \pm}\end{array}$ & 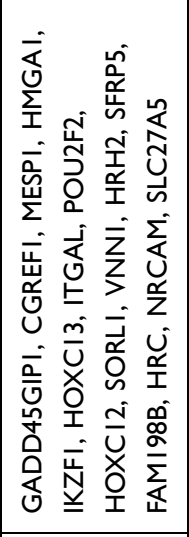 & 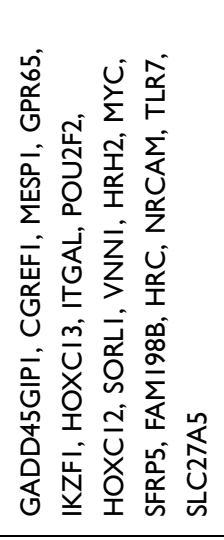 & 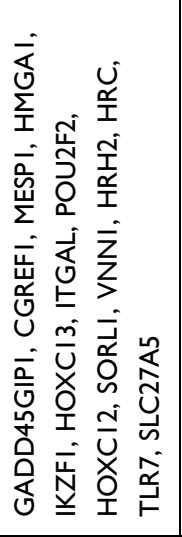 & 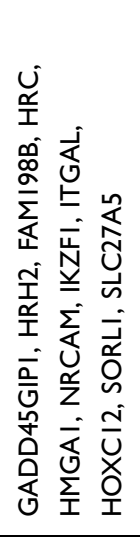 & 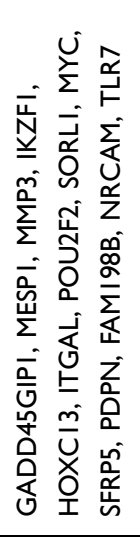 & 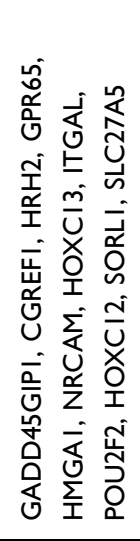 & 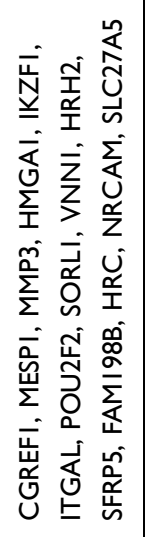 & 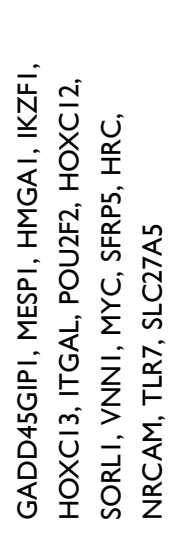 \\
\hline 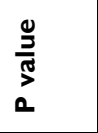 & $\begin{array}{l}\text { స్రి } \\
\text { ठํ. } \\
\text { ¿ }\end{array}$ & $\begin{array}{l}\text { م. } \\
\text { o. } \\
\stackrel{\circ}{0} \\
0\end{array}$ & $\begin{array}{l}\hat{\hat{L}} \\
\frac{0}{0} \\
0 \\
0\end{array}$ & 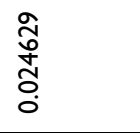 & 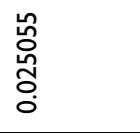 & 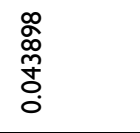 & 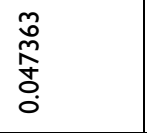 & 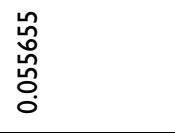 \\
\hline ஃீ & 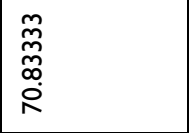 & 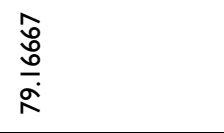 & นึ & 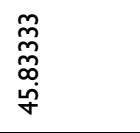 & $\underset{\substack{\underset{m}{m} \\
\infty}}{\substack{n \\
n}}$ & 오 & $\stackrel{\text { L }}{\mathrm{i}}$ & $\begin{array}{l}\hat{0} \\
. \\
0 \\
\stackrel{0}{0}\end{array}$ \\
\hline 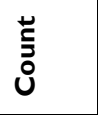 & $\simeq$ & $\underline{a}$ & $\underline{\underline{n}}$ & $=$ & \pm & $\simeq$ & $\underline{\underline{n}}$ & $\underline{\bullet}$ \\
\hline 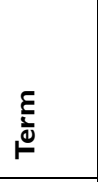 & 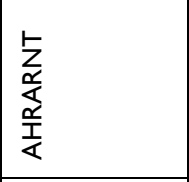 & $\frac{⿱ t}{\frac{1}{\alpha}}$ & $\begin{array}{l}\overline{\frac{\alpha}{\alpha}} \\
\frac{\alpha}{<}\end{array}$ & 娙 & $\underline{\underline{\tilde{O}}}$ & $\overline{\bar{Z}}$ & 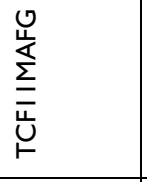 & $\bar{Z}_{\underline{I}}$ \\
\hline 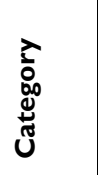 & 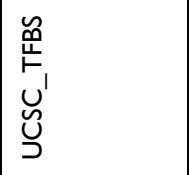 & 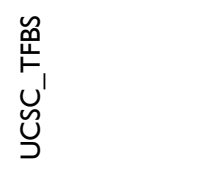 & 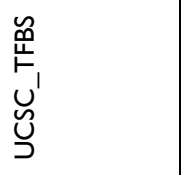 & 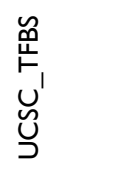 & 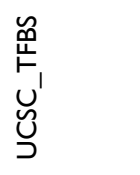 & 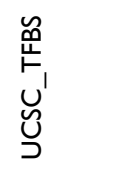 & 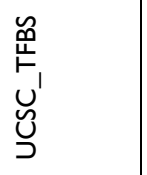 & 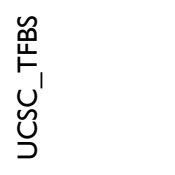 \\
\hline
\end{tabular}




\begin{tabular}{|c|c|c|c|c|c|}
\hline 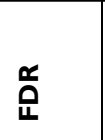 & $\begin{array}{l}\overline{\mathcal{Z}} \\
\text { ỗ } \\
\text { o. }\end{array}$ & 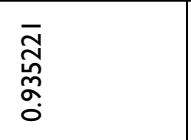 & 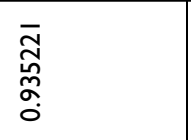 & 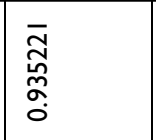 & 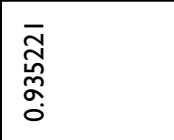 \\
\hline 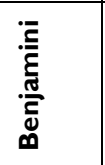 & $\begin{array}{l}\overline{\mathbb{Z}} \\
\text { o. } \\
\text { o. }\end{array}$ & 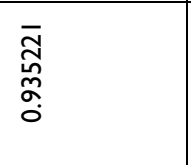 & 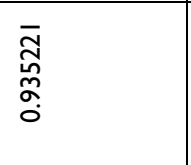 & 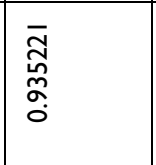 & 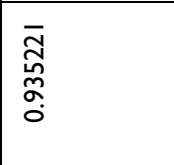 \\
\hline 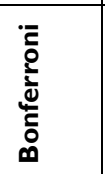 & 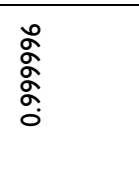 & - & - & - & - \\
\hline 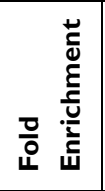 & 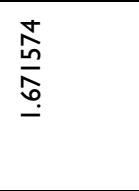 & 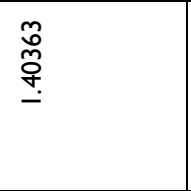 & 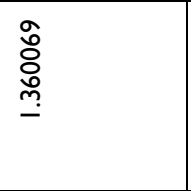 & $\begin{array}{l}\stackrel{o}{\bar{o}} \\
\underline{\underline{n}}\end{array}$ & 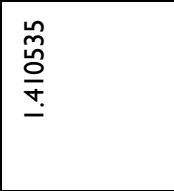 \\
\hline 옹 & $\begin{array}{l}\text { त̃ } \\
\text { î. }\end{array}$ & 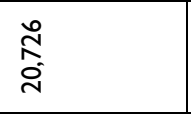 & 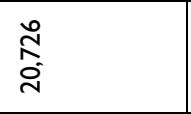 & 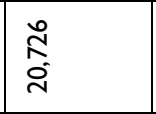 & 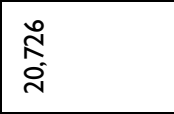 \\
\hline$\stackrel{g}{\frac{g}{I}}$ & 商 & 产 & $\begin{array}{l}\overline{\bar{o}} \\
\stackrel{\circ}{\circ}\end{array}$ & $\frac{\mathrm{g}}{n}$ & \begin{tabular}{l}
\multirow{2}{*}{} \\
\multirow{2}{*}{}
\end{tabular} \\
\hline 产 $\bar{g}$ & ก & $\tilde{\nu}$ & $\tilde{N}$ & $\tilde{\pi}$ & $\dddot{\sim}$ \\
\hline 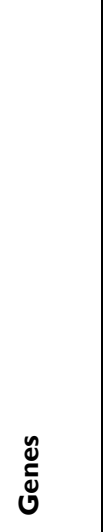 & 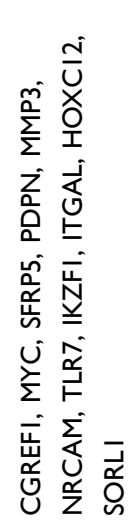 & 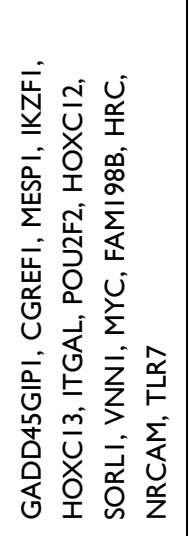 & 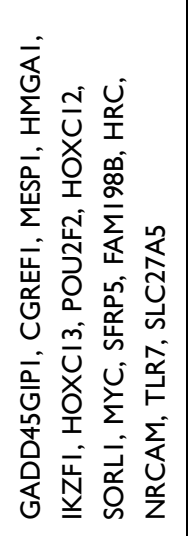 & 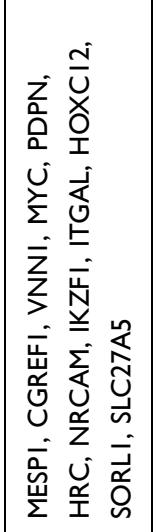 & 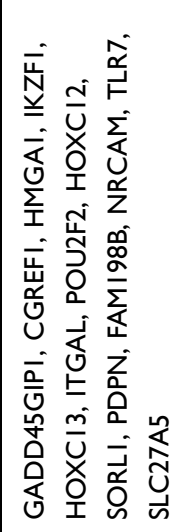 \\
\hline 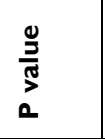 & 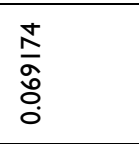 & $\begin{array}{l}\text { ờ } \\
\text { Oे̀ } \\
o \\
0\end{array}$ & $\begin{array}{l}\frac{\tilde{\alpha}}{\alpha} \\
\frac{\alpha}{\alpha o} \\
0 \\
0\end{array}$ & 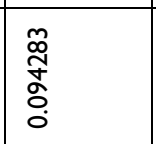 & 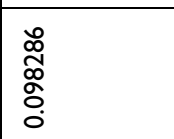 \\
\hline ஃீ & 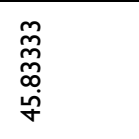 & นి & $\begin{array}{l}\hat{\circ} \\
\stackrel{\circ}{\circ} \\
\stackrel{0}{0} \\
0\end{array}$ & in & 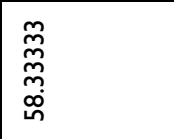 \\
\hline 䓂 & $=$ & $\underline{\underline{n}}$ & $\underline{\varrho}$ & $\simeq$ & \pm \\
\hline 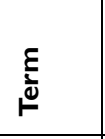 & 苫 & 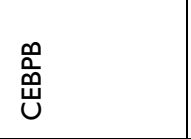 & 守 & 竞 & 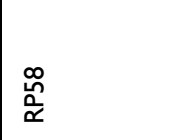 \\
\hline 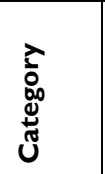 & 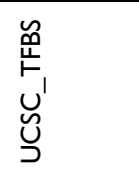 & 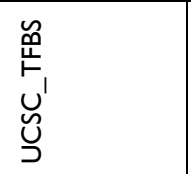 & 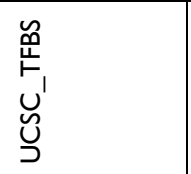 & 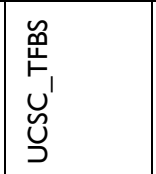 & 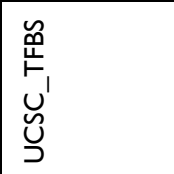 \\
\hline
\end{tabular}



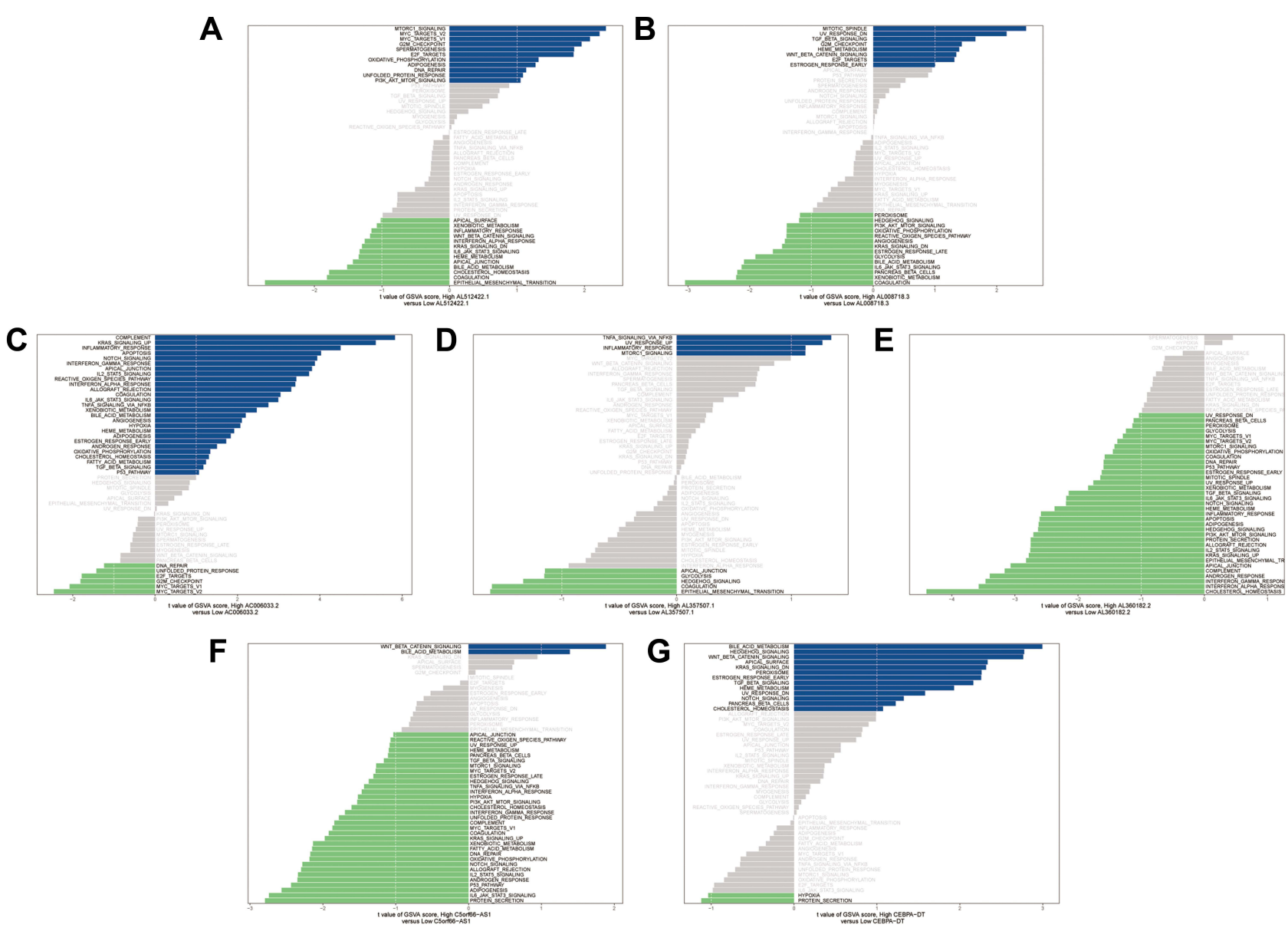

Figure 5 GSVA enrichment analysis of seven prognosis-related IncRNAs. (A) AL5I2422.1. (B) AL0087I8.3. (C) AC006033.2. (D) AL357507.I. (E) AL360I82.2. (F) C5orf66-ASI. (G) CEBPA-DT.
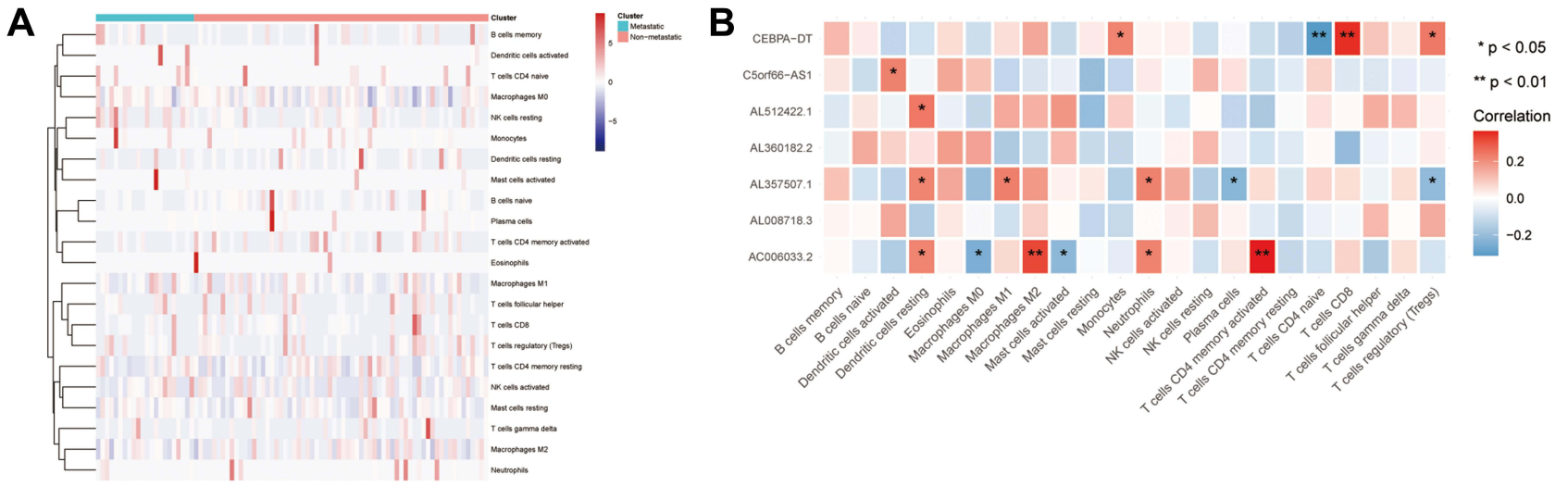

Figure 6 LncRNA and immune analysis. (A) Relationship between high/low group and immune cells; (B) Relationship between seven prognosis-related IncRNAs and immune cells. $*<0.05, * *<0.01$.

a high OS metastasis rate, while the other four had a low OS metastasis rate (Figure 7). AL512422.1 was found to be highly expressed in the U2OS and SJSA-1 cell lines than MNNG (Figure 7A). AL360182.2 and C5orf66-AS1 mRNA levels were found to be higher in four weakly metastatic cell lines (Figure 7E and F). However, AL008718.3 mRNA level was significantly downregulated in USO2, SAOS-2, SJSA-1, and HOS cell lines (Figure 7B). In addition, the mRNA level of AL357507.1 was only up-regulated in the SAOS-2 cell line 


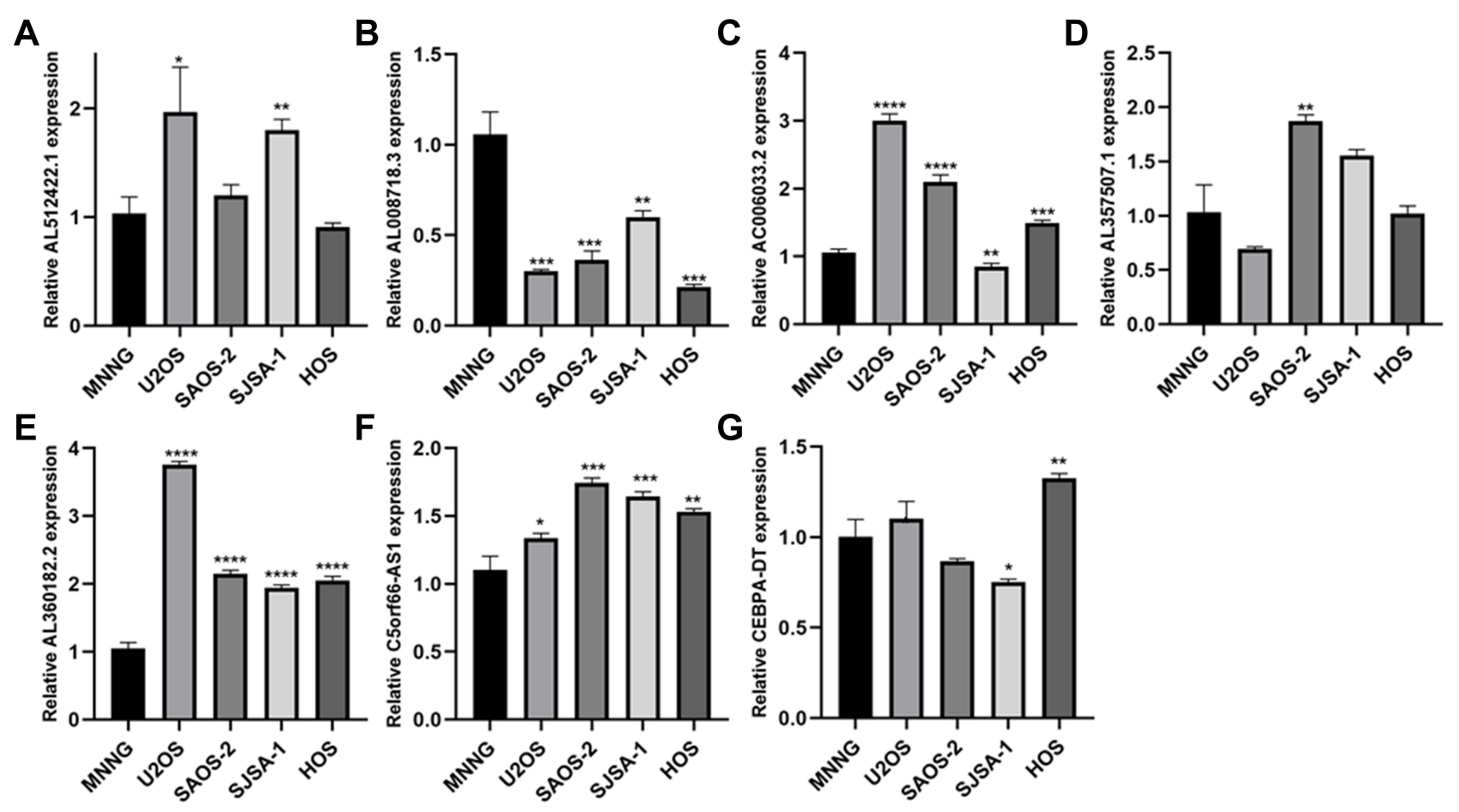

Figure 7 The qPCR result of seven IncRNAs in five OS highly metastasis and weakly metastasis cell lines. (A) AL5I2422.I. (B) AL0087I8.3. (C) AC006033.2. (D) AL357507.1. (E) AL360182.2. (F) C5orf66-ASI. (G) CEBPA-DT. $*<0.05, * *<0.01$, $* * *<0.001$, $* * * * 0.0001$.

(Figure 7D). Interestingly, even though they were all weak metastatic cell lines, the expression pattern differed. The mRNA level of AC006033.2 was higher in U2OS, SAOS2 , and HOS cell lines, and lower in SJSA-1 cell lines (Figure 7C). In the case of CEBPA-DT, the mRNA levels were higher in the HOS cell line and lower in the SJSA-1 cell line (Figure 7G).

\section{Discussion}

OS is an aggressive malignancy with poor outcomes, particularly in patients with metastases. ${ }^{29}$ The development of advanced treatments has resulted in significant improvement in the overall survival of patients with OS. Surgery is still the cornerstone of primary tumor treatment, whereas adjuvant chemotherapy is the most significant predictor of prognosis in patients with metastases. ${ }^{30,31}$ Chemotherapies, such as high-dose methotrexate, doxorubicin, cisplatin, and ifosfamide/etoposide are the most effective agents. However, the incidence of metastasis remains high in a significant proportion of OS patients. ${ }^{31}$ Therefore, to predict or inhibit the metastatic process, it is essential to identify targets to provide potential diagnostic and therapeutic strategies. ${ }^{32}$

Accumulating evidence suggests that lncRNAs are closely associated with tumorigenesis and progression.
IncRNAs play an essential role in a variety of biological processes implicated in carcinogenesis, including cell proliferation, migration, and tumor metastasis. ${ }^{33-36}$ Identifying an effective lncRNA biomarker may aid in the prevention of metastasis. For example, mtlncRNA LIPCAR is a novel biomarker for cardiac remodeling that can predict the survival rate of heart failure patients. ${ }^{37}$ Immunoreactive mice showed that targeted therapy of ASncmtRNAs could prevent lung metastases following melanoma resection. ${ }^{38}$ The lentiviral construct targeting ASncmtRNAs could inhibit B16F10 primary tumor proliferation. ${ }^{39}$ In vivo studies in isogenic mouse renal cancer models revealed that ASncmtRNAs-targeting ASOs could completely reverse tumor growth. ${ }^{40}$ In summary, lncRNA targeted therapy may be a potential therapeutic approach for improving prognosis.

In this study, we established a new risk-score model based on prognosis-related lncRNA generated from a series of bioinformatics analyses to predict the metastasis of OS patients. Based on their median risk score, all patients were classified as high or low-risk groups. In comparison to the low-risk group, patients in the high group had a poor survival rate. By calculating the AUC value, this model exhibited a strong prediction capacity. The co-expression network revealed an association between $\mathrm{TF}$ and 24 
prognosis-related mRNAs, with the seven lncRNAs having the closest relationship. Zhong et al reported that HSF2 was involved in the growth and metastasis of lung cancer. ${ }^{41}$ It is important to note that the most common site of OS metastasis in the lungs and lung metastases are associated with poor prognosis. ${ }^{30}$ NRF2 is a transcription factor that modulates the level of reactive oxygen species (ROS), detoxifying agents, and antioxidants in the body. The increase in NRF2 in the nuclei is closely related to bone metastases in OS patients. ${ }^{42}$ Furthermore, Cindy et al revealed that catecholamines and their receptors could be potential molecular markers for OS progression. ${ }^{43}$ The catecholamine metabolic process and catechol-containing compound metabolic process were reported as a consequence of 24 prognosis-related mRNA enrichment.

Several overlapping pathways were discovered among the seven lncRNAs enrichment findings, confirming the critical biological function of the seven lncRNAs. The terms "NOTCH_SIGNALING", "HYPOXIA", "WNT_ BETA_CATENIN_SIGNALING", and "HEDGEHOG_ SIGNALING" appeared more often. Several studies have shown that the Notch pathway regulates OS metastasis and may be useful for therapeutic targeting in the near future. $^{44,45}$ Fang et al reported that the Notch pathway could be pharmacologically inhibited, which was a huge step in targeted therapeutic strategies. ${ }^{46}$ CNOT1, in collaboration with LMNA, may exacerbate OS tumorigenesis through the Hedgehog signaling pathway. ${ }^{47}$ In addition, the hypoxia-HIF-1 $\alpha$-CXCR4 pathway plays a critical role during the migration of human OS cells. ${ }^{48}$ Furthermore, NRP2 was shown to be overexpressed in OS cell lines; depleting NRP2 through downregulation of the active Wnt-signaling pathway would significantly reduce the tumor burden and metastasis rate in OS cell lines. ${ }^{49}$ Additionally, we found that three of the seven lncRNAs were associated with resting dendritic cells. According to Jones et al, IL23 expression and monocyte-derived dendritic cells have both activating and suppressive effects on cytotoxic T cells, as well as direct effects on osteoblasts or associated osteoclasts. ${ }^{50}$ It is worth mentioning that under hypoxic conditions, dendritic cells secrete significant amounts of osteopontin, which contributes to tumor metastasis. $^{51}$

There were some limitations in the present study. One limitation is the relatively small sample size, even though recruitment is still ongoing. Another limitation is that no additional analysis stratified by age was conducted to account for metastasis. In conclusion, we established a novel model with excellent reliability and accuracy for predicting the metastasis of OS patients.

\section{Abbreviation}

AUC, area under receiver operating characteristic curve; $\mathrm{BP}$, biological processes; CC, cellular components; EMT, epithelial-mesenchymal transition; FDR, false discovery rate; GSEA, Gene set enrichment analysis; GSVA, Gene Set Variation Analysis; KM, Kaplan-Meier; LASSO, least absolute shrinkage and selection operator; lncRNAs, long non-coding RNAs; MsigDB, Molecular Signatures Database; OS, osteosarcoma; ROS, reactive oxygen species; TF, transcription factor; Tregs, T cells regulatory.

\section{Data Sharing Statement}

Publicly available datasets from the TARGET datasets (https://ocg.cancer.gov/programs/target) were analyzed in this study. The datasets generated during and/or analyzed during the current study are available from the corresponding author upon reasonable request.

\section{Ethics Statement}

The study was approved by the Ethical Committee of the Affiliated Hospital of Qingdao University and experiments were performed in accordance with the Ethical Committee's guidelines and regulations. The study complies with guidelines by the Declaration of Helsinki.

\section{Author Contributions}

Zhang H, Chen GH and Lyu CY designed the study, acquired the data, and wrote the manuscript. $\mathrm{Xu} \mathrm{Y}$ reviewed the manuscript. Lyu $\mathrm{XJ}$ and Rong $\mathrm{C}$ contributed to the Statistical analysis and graphing. Wang YZ and Lyu CY supervised the study. Zhang $\mathrm{H}$ and Chen $\mathrm{GH}$ contribute equally to this paper. All authors made a significant contribution to the work reported, whether that is in the conception, study design, execution, acquisition of data, analysis and interpretation, or in all these areas; took part in drafting, revising or critically reviewing the article; gave final approval of the version to be published; have agreed on the journal to which the article has been submitted; and agree to be accountable for all aspects of the work.

\section{Funding}

This study was supported by National Natural Science Foundation of China (Grant No.81802151), Shandong Provincial Natural Science Foundation of China (Grant No. ZR2016HQ05; ZR2017BH089; ZR2019MH012), 
General Funding of China Postdoctoral Science Foundation (Grant No.2018M642616) and Qingdao Applied Basic Research Youth Special Project (Grant No.19-6-2- 55-cg).

\section{Disclosure}

Authors declare that they have no conflict of interest.

\section{References}

1. Dorfman HD, Czerniak BJ. Bone cancers. Cancer. 1995;75 (S1):203-210.

2. Mirabello L, Troisi RJ, Savage SA. Osteosarcoma incidence and survival rates from 1973 to 2004: data from the Surveillance, Epidemiology, and End Results Program. Cancer. 2009;115 (7):1531-1543. doi:10.1002/cncr.24121

3. Unni KK, Inwards CY. Dahlin's Bone Tumors: General Aspects and Data on 10,165 Cases. Lippincott Williams \& Wilkins; 2010.

4. Larsson SE, Lorentzon RJ. The incidence of malignant primary bone tumours in relation to age, sex and site: a study of osteogenic sarcoma, chondrosarcoma and Ewing's sarcoma diagnosed in Sweden from 1958 to 1968. J Bone Joint Surg Br. 1974;56 (3):534-540. doi:10.1302/0301-620X.56B3.534

5. Polednak AP. Primary bone cancer incidence in black and white residents of New York State. Cancer. 1985;55(12):2883-2888. doi:10.1002/1097-0142(19850615)55:12<2883::AIDCNCR2820551231>3.0.CO;2-Q

6. Ji J, Hemminki K. Familial risk for histology-specific bone cancers: an updated study in Sweden. Eur J Cancer. 2006;42(14):2343-2349. doi:10.1016/j.ejca.2005.11.043

7. Bacci G, Longhi A, Fagioli F, et al. Adjuvant and neoadjuvant chemotherapy for osteosarcoma of the extremities: 27 year experience at Rizzoli Institute, Italy. Cancer. 2005;41(18):2836-2845.

8. Link MP, Goorin AM, Miser AW, et al. The effect of adjuvant chemotherapy on relapse-free survival in patients with osteosarcoma of the extremity. N Engl J Med. 1986;314(25):1600-1606.

9. Rosen G, Caparros B, Huvos AG, et al. Preoperative chemotherapy for osteogenic sarcoma: selection of postoperative adjuvant chemotherapy based on the response of the primary tumor to preoperative chemotherapy. Cancer. 1982;49(6):1221-1230. doi:10.1002/ 1097-0142(19820315)49:6<1221::AID-CNCR2820490625>3.0. $\mathrm{CO} ; 2-\mathrm{E}$

10. Dong J, Liu Y, Liao W, et al. miRNA-223 is a potential diagnostic and prognostic marker for osteosarcoma. J Bone Oncol. 2016;5 (2):74-79. doi:10.1016/j.jbo.2016.05.001

11. Wang M, Xie R, Si H, et al. Integrated bioinformatics analysis of miRNA expression in osteosarcoma. Artif Cells Nanomed Biotechnol. 2017;45(5):936-943. doi:10.1080/21691401.2016.1196456

12. Di Fiore R, Drago-Ferrante R, Pentimalli F, et al. Let-7d miRNA shows both antioncogenic and oncogenic functions in osteosarcomaderived 3AB-OS cancer stem cells. J Cell Physiol. 2016;231 (8):1832-1841. doi:10.1002/jcp.25291

13. Schwartz CL, Gorlick R, Teot L, et al. Multiple drug resistance in osteogenic sarcoma: INT0133 from the Children's Oncology Group. $J$ Clin Oncol. 2007;25(15):2057-2062. doi:10.1200/JCO.2006.07. 7776

14. Gill J, Ahluwalia MK, Geller D, et al. New targets and approaches in osteosarcoma. Pharmacol Ther. 2013;137(1):89-99. doi:10.1016/j. pharmthera.2012.09.003

15. Shi J, Dong B, Cao J, et al. Long non-coding RNA in glioma: signaling pathways. Oncotarget. 2017;8(16):27582. doi:10.18632/ oncotarget. 15175
16. Idogawa M, Ohashi T, Sasaki Y, et al. Long non-coding RNA NEAT1 is a transcriptional target of p53 and modulates p53-induced transactivation and tumor-suppressor function. Int $J$ Cancer. 2017;140 (12):2785-2791. doi:10.1002/ijc.30689

17. Liang Y, Zhang D, Zheng T, et al. IncRNA-SOX2OT promotes hepatocellular carcinoma invasion and metastasis through miR-1225p-mediated activation of PKM2. Oncogenesis. 2020;9(5):54. doi:10.1038/s41389-020-0242-z

18. Gooding AJ, Zhang B, Jahanbani FK, et al. The lncRNA BORG Drives Breast Cancer Metastasis and Disease Recurrence. Sci Rep. 2017;7(1):12698. doi:10.1038/s41598-017-12716-6

19. Wang Z, Yang B, Zhang M, et al. IncRNA Epigenetic Landscape Analysis Identifies EPIC1 as an Oncogenic lncRNA that Interacts with MYC and Promotes Cell-Cycle Progression in Cancer. Cancer Cell. 2018;33(4):548.

20. Kitamura T, Qian BZ, Soong D, et al. CCL2-induced chemokine cascade promotes breast cancer metastasis by enhancing retention of metastasis-associated macrophages. J Exp Med. 2015;212 (7):1043-1059. doi:10.1084/jem.20141836

21. Li C, Wang S, Xing Z, et al. A ROR1-HER3-IncRNA signalling axis modulates the Hippo-YAP pathway to regulate bone metastasis. Nat Cell Biol. 2017;19(2):106-119. doi:10.1038/ncb3464

22. Ji P, Diederichs S, Wang W, et al. MALAT-1, a novel noncoding RNA, and thymosin $\beta 4$ predict metastasis and survival in early-stage non-small cell lung cancer. Oncogene. 2003;22(39):8031-8041. doi:10.1038/sj.onc. 1206928

23. Shen L, Chen L, Wang Y, et al. Long noncoding RNA MALAT1 promotes brain metastasis by inducing epithelial-mesenchymal transition in lung cancer. J Neurooncol. 2015;121(1):101-108. doi:10. 1007/s11060-014-1613-0

24. Ying L, Chen Q, Wang Y, et al. Upregulated MALAT-1 contributes to bladder cancer cell migration by inducing epithelial-to-mesenchymal transition. Mol Biosyst. 2012;8(9):2289-2294. doi:10.1039/c2mb $25070 \mathrm{e}$

25. Fan Y, Shen B, Tan M, et al. TGF- $\beta$-induced upregulation of malat1 promotes bladder cancer metastasis by associating with suz12. Clin Cancer Res. 2014;20(6):1531-1541. doi:10.1158/1078-0432.CCR13-1455

26. Kim K, Jutooru I, Chadalapaka G, et al. HOTAIR is a negative prognostic factor and exhibits pro-oncogenic activity in pancreatic cancer. Oncogene. 2013;32(13):1616-1625. doi:10.1038/onc.2012. 193

27. Zhou Y, Zhang X, Klibanski A. MEG3 noncoding RNA: a tumor suppressor. J Mol Endocrinol. 2012;48(3):R45-R53. doi:10.1530/ JME-12-0008

28. Peng W, Si S, Zhang Q, et al. Long non-coding RNA MEG3 functions as a competing endogenous RNA to regulate gastric cancer progression. J Mol Endocrinol. 2015;34(1):1-10.

29. Hou CH, Lin FL, Hou SM, et al. Cyr61 promotes epithelial-mesenchymal transition and tumor metastasis of osteosarcoma by Raf-1/MEK/ERK/Elk-1/TWIST-1 signaling pathway. Mol Cancer. 2014;13(1):1-13. doi:10.1186/1476-4598-13-236

30. Geller DS, Gorlick R. Osteosarcoma: a review of diagnosis, management, and treatment strategies. Clin Adv Hematol Oncol. 2010;8 (10):705-718.

31. Ferguson WS, Goorin AM. Current treatment of osteosarcoma. Cancer Invest. 2001;19(3):292-315. doi:10.1081/CNV-100102557

32. He M, Wang G, Jiang L, et al. miR-486 suppresses the development of osteosarcoma by regulating PKC- $\delta$ pathway. Int J Oncol. 2017;50 (5):1590-1600. doi:10.3892/ijo.2017.3928

33. Li G, Zhang H, Wan X, et al. Long noncoding RNA plays a key role in metastasis and prognosis of hepatocellular carcinoma. Biomed Res Int. 2014;2014:780521.

34. Maass PG, Luft FC, Bähring S. Long non-coding RNA in health and disease. J Mol Med (Berl). 2014;92(4):337-346. doi:10.1007/s00109014-1131-8 
35. Tripathi V, Shen Z, Chakraborty A, et al. Long noncoding RNA MALAT1 controls cell cycle progression by regulating the expression of oncogenic transcription factor B-MYB. PLoS Genet. 2013;9(3): e1003368. doi:10.1371/journal.pgen.1003368

36. Zhang A, Xu M, Mo YY. Role of the lncRNA-p53 regulatory network in cancer. J Mol Cell Biol. 2014;Jun(3):181-191. doi:10.1093/ $\mathrm{jmcb} / \mathrm{mju} 013$

37. Kumarswamy R, Bauters C, Volkmann I, et al. Circulating long noncoding RNA, LIPCAR, predicts survival in patients with heart failure. Circ Res. 2014;114(10):1569-1575. doi:10.1161/ CIRCRESAHA.114.303915

38. Lobos-González L, Silva V, Araya M, et al. Targeting antisense mitochondrial ncRNAs inhibits murine melanoma tumor growth and metastasis through reduction in survival and invasion factors. Oncotarget. 2016;7(36):58331. doi:10.18632/oncotarget.11110

39. Varas-Godoy M, Lladser A, Farfan N, et al. In vivo knockdown of antisense non-coding mitochondrial RNA s by a lentiviral-encoded sh RNA inhibits melanoma tumor growth and lung colonization. Pigment Cell Melanoma Res. 2018;31(1):64-72. doi:10.1111/pcmr.12615

40. Borgna V, Villegas J, Burzio VA, et al. Mitochondrial ASncmtRNA-1 and ASncmtRNA-2 as potent targets to inhibit tumor growth and metastasis in the RenCa murine renal adenocarcinoma model. Oncotarget. 2017;8(27):43692. doi:10.18632/oncotarget.18460

41. Zhong YH, Cheng HZ, Peng H, et al. Heat shock factor 2 is associated with the occurrence of lung cancer by enhancing the expression of heat shock proteins. Oncol Lett. 2016;12(6):5106-5112. doi: $10.3892 / \mathrm{ol} .2016 .5368$

42. Zhang J, Wang X, Wu W, et al. Expression of the Nrf2 and Keap1 proteins and their clinical significance in osteosarcoma. Biochem Biophys Res Commun. 2016;473(1):42-46. doi:10.1016/j.bbrc.2016.03.047

43. Bandala C, Ávila-luna A, Gómez-López M, et al. Catecholamine levels and gene expression of their receptors in tissues of adults with osteosarcoma. Arch Physiol Biochem. 2019;10:1-7.
44. Tanaka M, Setoguchi T, Hirotsu M, et al. Inhibition of Notch pathway prevents osteosarcoma growth by cell cycle regulation. Br J Cancer. 2009;100(12):1957-1965. doi:10.1038/sj.bjc.6605060

45. Dai G, Liu G, Zheng D, et al. Inhibition of the Notch signaling pathway attenuates progression of cell motility, metastasis, and epithelial-to-mesenchymal transition-like phenomena induced by low concentrations of cisplatin in osteosarcoma. Eur J Pharmacol. 2021;15(899):174058. doi:10.1016/j.ejphar.2021.174058

46. Fang L, Li B, Yu D, et al. Analysis of changes in the expression of Notch1 and HES1 and the prognosis of osteosarcoma patients following surgery. Oncol Lett. 2020;20(4):1. doi:10.3892/ol.2020.12156

47. Cheng D, Li J, Li S, et al. CNOT 1 cooperates with LMNA to aggravate osteosarcoma tumorigenesis through the Hedgehog signaling pathway. Mol Oncol. 2017;11(4):388-404. doi:10.1002/18780261.12043

48. Guo M, Cai C, Zhao G, et al. Hypoxia promotes migration and induces CXCR4 expression via HIF-1 $\alpha$ activation in human osteosarcoma. PLoS One. 2014;9(3):e90518. doi:10.1371/journal.pone.00 90518

49. Ji T, Guo Y, Kim K, et al. Neuropilin-2 expression is inhibited by secreted Wnt antagonists and its down-regulation is associated with reduced tumor growth and metastasis in osteosarcoma. Mol Cancer. 2015;14(1):1-14. doi:10.1186/s12943-015-0359-4

50. Jones KB. Dendritic Cells Drive Osteosarcomagenesis through Newly Identified Oncogene and Tumor Suppressor. Cancer Discov. 2019;9(11):1484-1486. doi:10.1158/2159-8290.CD-19-0994

51. Yang M, Ma C, Liu S, et al. Hypoxia skews dendritic cells to a $\mathrm{T}$ helper type 2-stimulating phenotype and promotes tumour cell migration by dendritic cell-derived osteopontin. Immunology. 2009;128 (1pt2):e237-e249. doi:10.1111/j.1365-2567.2008.02954.x

\section{Publish your work in this journal}

Cancer Management and Research is an international, peer-reviewed open access journal focusing on cancer research and the optimal use of preventative and integrated treatment interventions to achieve improved outcomes, enhanced survival and quality of life for the cancer patient.
The manuscript management system is completely online and includes a very quick and fair peer-review system, which is all easy to use. Visit http://www.dovepress.com/testimonials.php to read real quotes from published authors. 